Xiaohua Ni • Yushu Ma • Haipeng Cheng • Min Jiang

Lingchen Guo • Chaoneng Ji • Shaohua Gu

Yueqong Cao $\cdot$ Yi Xie $\cdot$ Yumin Mao

\title{
Molecular cloning and characterization of a novel human Rab (Rab2B) gene
}

\begin{abstract}
Rab proteins are small-molecular-weight guanosine triphosphatases (GTPases) that control vesicular traffic in eukaryotic cells. The small GTPase Rab2 is a resident of pre-Golgi intermediates and is required for protein transport from the endoplasmic reticulum to the Golgi complex. We identified a novel human Rab $(\operatorname{Rab} 2 B)$ gene that was $2312 \mathrm{bp}$ in length and encoded a protein of 216 amino acid residues. The protein shared high homology with mouse Rab2 (identity $83 \%$, similarity $91 \%$ ). The expression pattern of the human $R a b 2 B$ gene showed that there is a transcript in kidney, prostate, lung, liver, thymus, colon, pancreas, and skeletal muscle, and low levels in placenta, whereas specific bands of the transcript could not be detected in heart, brain, spleen, testis, ovary, small intestine, and leukocyte. Overexpression has been observed in colon adenocarcinoma CX-1. The Rab2B gene consists of nine exons and eight introns and is mapped to chromosome 14q11.1-14q11.2 by bioinformatics analysis.
\end{abstract}

Key words $R a b 2 B$. MTC panel PCR - Chromosome 14q11.1-14q11.2 $\cdot$ Tumor $\cdot$ Colon adenocarcinoma

The Ras superfamily, which includes more than 100 members, has been grouped into four subfamilies: Ras-encoded proteins, the part of signal transduction pathways that lead to cell prolifeation and differentiation in response to external signals; Rho-encoded proteins that are involved in cytoskeleton organization; ADP ribosylation factor proteins; and Rab proteins.

The Rab oncogene family of small guanosine triphosphatases (GTPases) regulates vesicular traffic between specific compartments of the endocytic and exocytic pathways of eukaryotic cells (Nuoffer et al. 1994) and behaves as a

X. Ni $\cdot$ Y. Ma $\cdot$ H. Cheng $\cdot$ M. Jiang $\cdot$ L. Guo $\cdot$ C. Ji $\cdot$ S. Gu $\cdot$ Y. Cao Y. Xie · Y. Mao $(\bowtie)$

State Key Laboratory of Genetic Engineering, Institute of Genetics, School of Life Sciences, Fudan University, Shanghai 200433, P.R.

China

Tel. +86-21-65643573; Fax +86-21-65642502

e-mail: ymmao@fudan.edu.cn membrane-associated molecular switch to regulate budding, transport, and fusion reactions in vesicular transport. More than 50 Rab proteins have been described in mammalian cells, each with a specific subcellular localization and many with specific patterns of tissue distribution (PereiralLeal and Seabra 2001). Rab proteins, like other members of the Ras superfamily, contain four highly conserved sequence motifs required for guanine nucleotide binding (Bourne et al. 1991). The small GTPase Rab2 is a resident of pre-Golgi intermediates and required for protein transport from the endoplasmic reticulum (ER) to the Golgi complex (Tisdale et al. 1992). It also contains conserved GTP-binding domains as well as hypervariable carboxyterminal and amino-terminal domains.

During our large-scale sequencing analysis of human cDNA libraries, we cloned a full-length cDNA of the human $R a b 2 B$ gene, encoding a homologue of the mouse $R a b 2$ gene and human $R a b 2 A$ gene. The nucleotide sequence has been submitted to the Genbank/EMBL Database with accession number AF468652 (AF091029 in GenBank is a partial sequence of Rab2B). The cDNA consists of $2312 \mathrm{bp}$ and contains an open reading frame (ORF) of $651 \mathrm{bp}$ encoding a protein of 216 amino acids. Domain analysis using the Web service of the National Center for Biotechnology Information (NCBI) (RPS-BLAST) showed that the Rab domain is located at residues 7-167 of the protein sequence $(100.0 \%$ aligned). The predicted protein also contains the double cysteine prenylation motif at the $\mathrm{C}$ terminus. The cDNA is considered to be full length because there is an upstream in-frame stop codon (TGA) and a polyA signal (AATAAA) after the ORF. Bioinformatics analysis using BLASTx revealed that Rab2B shared a high degree of homology with mouse Rab2 (mRab2, 83\% identity and $91 \%$ similarity) and human Rab2A (hRab2A, $82 \%$ identity and $89 \%$ similarity). Various levels of homology with rabbit Rab2 (rRab2) and chicken Rab2 (cRab2) were also detected (Fig. 1).

To determine the chromosomal localization of the human $R a b 2 B$ gene, we used an international human genome database at NCBI. The gene was mapped to contig NT-019583, spanning $17942 \mathrm{bp}$. The contig was located at 
Fig. 1. Alignment of human $R a b 2 B$ with hRab2A, mRab2, rRab2, and cRab2. Numbers on the right refer to the last amino acid in each corresponding line. Identity is indicated by a black box, and similarity is indicated by a gray box
hRab2B : hRab2A : mRab2

rRab2 cRab2

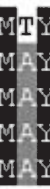
YAY LE KYI I I GT GVGKSCLILQE T DKREQPVHDLT I GVEE GARMV AYLFKYIIIGDTGVGK SCLILQF T DKRE Q PVHDLT I GVEE GARMI YAY LE KYIIIGDT GVGK SCLLLQET DKREQPVHDLT I GVEE GARMI YAY LE KYIIIGDT GVGK SCLLLQE T DKREQPVHDLT IGVEE GARMII YAY LEKYIIIGDT GVGK SCLLLQET DKREQPVHDLT I GVEE GARMI I
50

50

50

50

50

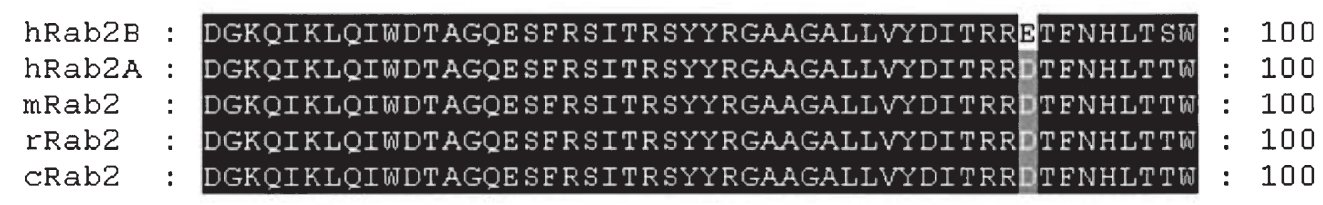

hRab2B : LEDARQHSSSNMVIMLIGNKSDLESRRDVKREEGEAEAREHGLIFMETSA : 150 hRab2A : LEDARQHSN SNMVIMLIGNKSDLESRR VVKKEEGEAEAREHGLMFMET SA : 150 mRab2 : LEDARQHSN SNMVIMLIGNKSDLESRR SVKKEEGEAEAREHGLIEMET SA : 150 rRab2 : LEDARQH SN SNMVIMLIGNKSDLESRREVKKEEGEAEAREHGLIFMET SA : 150 CRab2 : LEDARQH SN SNMVIMLIGNKSDLESRR VVKKEEGEAEAREHGLIFMETSA : 150

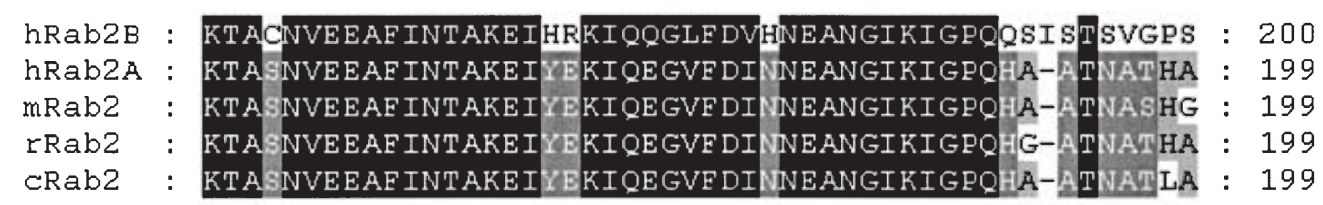

Table 1. Nucleotide sequence of exon-intron junctions of the human $\operatorname{Rab} 2 \mathrm{~B}$ gene

\begin{tabular}{|c|c|c|c|c|c|}
\hline $3^{\prime}$ Splice acceptor & Exon & Size (bp) & 5' Splice donor & Intron & Size (bp) \\
\hline cDNAend AAGAAGAAGATC & 1 & 139 & TCGGAGACACAGgtaaccetcagg & 1 & 227 \\
\hline tttctcttcag GTGTGGGGAAGT & 2 & 72 & ACCTCACAATAGgtaagtgcatct & 2 & 1593 \\
\hline ctttaccgacagGTGTGGAGTTTA & 3 & 68 & ATCTGGGATACGgtgagagtacaa & 3 & 6114 \\
\hline ttggtgggaaagGCTGGGCAAGAA & 4 & 82 & CGACATTACAAGgtgagccagatc & 4 & 220 \\
\hline tttctgctgtagGCGTGAAACCTT & 5 & 94 & TGGGAATAAGAGgtaaaattgtat & 5 & 4589 \\
\hline aatctcttgcagTGACCTAGAGTC & 6 & 112 & AATGTTGAAGAGgtactatttgga & 6 & 1237 \\
\hline tgtctctttcagGCCTTCATTAAC & 7 & 69 & GTCCACAATGAGgtaagaaagggt & 7 & 1046 \\
\hline tttcttttacagGCAAATGGCATC & 8 & 357 & GTTAGGATCAAAgttgattatcac & 8 & 605 \\
\hline cctcccaaagTGCTGGATTACA & 9 & 1318 & GCATGTATCAAA & 9 & \\
\hline
\end{tabular}

Intron sequence shown in lowercase and exon sequence in uppercase letters

14q11.1-14q11.2, whereas Rab2A was located at 8q12.1. Comparing our cDNA with the genome sequence of $R a b 2 B$ suggested that the gene consisted of nine exons and eight introns. All sequences at the exon-intron junctions were consistent with the AG-GT rule (Table 1).

The tissue distribution of $R a b 2 B$ was determined by multiple tissue cDNA (MTC) Panel polymerase chain reaction (PCR) and human tumor panel PCR. Two human MTC panels (Clontech, Palo Alto, CA, USA) and human tumor panels (Clontech) were used as PCR templates according to the manufacturer's protocol. The sequences for human $R a b 2 B$ specific primer pairs were $5^{\prime}$-ATTGTAG TGTCTTTCACCAGATAGACTTC-3' (Rab2B F, 1416$1444 \mathrm{bp}$ ) and 5'-GAGTAGCACTAGGCACTGTAAAG GAATG-3' (Rab2B R, 1833-1860bp). Thirty-five cycles of amplification $\left(30 \mathrm{~s}\right.$ at $94^{\circ} \mathrm{C}, 1 \mathrm{~min}$ at $65^{\circ} \mathrm{C}$, and $1 \mathrm{~min}$ at $72^{\circ} \mathrm{C}$ ) were performed using elongase DNA polymerase (GIBCO BRL, Gaithersburg, MD, USA). The PCR product of 


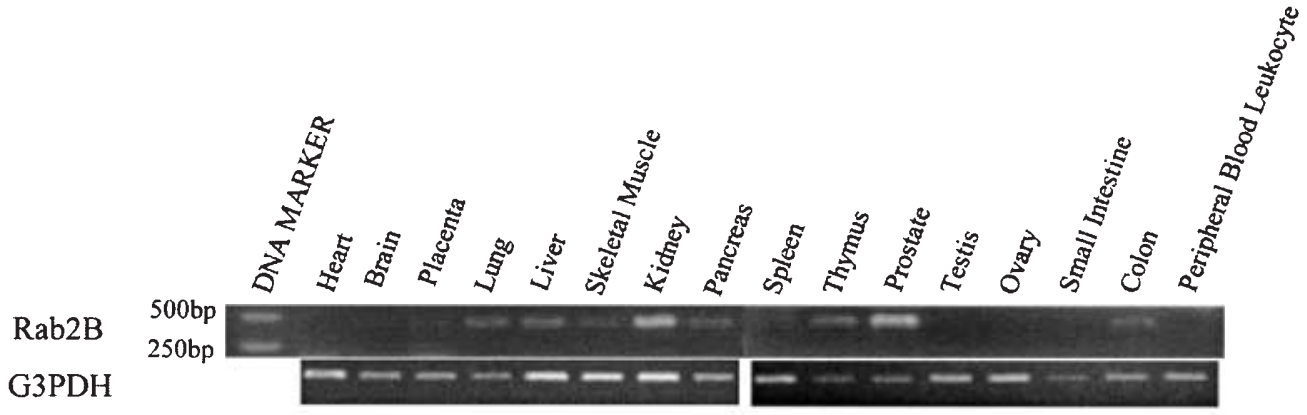

Rab2B

G3PDH

Fig. 2. Tissue distribution of $R a b 2 B$ expression in normal tissues and tumor tissues. Lane 1, DNA marker; lane 2, Pancreatic adenocarcinoma GI-103, a poorly differentiated carcinoma propagated from the ascitic fluid of a pancreatic adenocarcinoma; lane 3, ovarian carcinoma GI-102, an undifferentiated carcinoma isolated from a primary ovarian carcinoma; lane 4, colon adenocarcinoma GI-112, a moderately to poorly differentiated adenocarcinoma established from a 54-year-old woman; lane 5, prostatic adenocarcinoma PC3, a grade IV adenocarci-

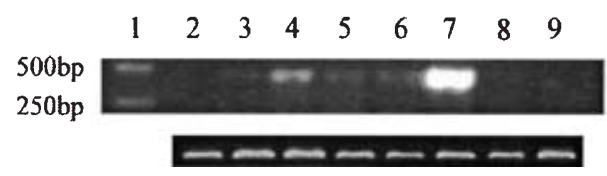

noma from a 65-year-old Caucasian; lane 6, lung carcinoma GI-117, a poorly differentiated carcinoma established from the tumor of a 62 year-old woman; lane 7, colon adenocarcinoma CX-1, a moderately well-differentiated adenocarcinoma consistent with gastrointestinal origin; lane 8 , lung carcinoma LX-1, a poorly differentiated carcinoma that is a surgical explant from metastasis from a 48-year-old man; lane 9 , breast carcinoma GI-101, a poorly differentiated mammary carcinoma isolated from recurrent ductal carcinoma

$R A B 2 B$ was then resolved on a $1.5 \%$ metaphor agarose gel (FMC, Philadelphia, PA, USA). In total, 16 human tissues and 8 human tumor tissues were tested (Fig. 2).

Earlier studies showed that the small GTPase Rab2 is a resident of pre-Golgi intermediates and required for protein transport from the ER to the Golgi complex. Most of the Rab proteins indentified so far are ubiquitously expressed in many tissues, although their level of expression may vary from one cell type to another. However, some Rab proteins have been found to be specific to cell type or tissue (Lutcke et al. 1993; Baldini et al. 1992).

Some data shows that, as an oncogene, the Rab2A protein is frequently overexpressed in peripheral blood mononuclear cells from patients with solid neoplasms. In addition, the expression is shown to be greatly modified during the course of therapy. This observation implies that a small GTP-binding protein associates with neoplastic diseases during immunological events (Culine et al. 1993, 1994). The Rab2A gene mRNA analysis in 70 tumor samples from various origins showed no obvious difference between malignant tissues and their normal counterparts. However, overexpression has been observed at the RNA or protein levels in peripheral blood mononuclear cells from nine patients with Sezary syndrome (Culine et al. 1992). Our data reveals that $R a b 2 B$ is overexpressed in colon adenocarcinoma CX-1 that is different from Rab2A.

We report here a novel human Rab $(R a b 2 B)$ gene, which is located at chromosome $14 \mathrm{q} 11.1-14 \mathrm{q} 11.2$, as shown by bioinformatics analysis from NCBI. Rab2A is located at $8 \mathrm{q} 12.1$. Rab2B is $2312 \mathrm{bp}$ in length and encodes a protein of 216 amino acid residues in which the Rab family domain is identified. By using Blastx from NCBI, we find that the predicted protein shares high homology with mouse Rab2

(83\% identity and $91 \%$ similarity) and human Rab2A (82\% identity and $89 \%$ similarity). The expression pattern of the human $R a b 2 B$ gene reveals a transcript in kidney, prostate, lung, liver, thymus, and colon, and a lower expression level in placenta, pancreas, and skeletal muscle. No specific bands of the transcript could be detected in heart, brain, spleen, testis, ovary, small intestine, and leukocyte. The human tumor panel showed a transcript in colon adenocarcinoma CX-1, colon adenocarcinoma GI-112, prostatic adenocarcinoma PC3, and lung carcinoma GI-117, and a lower level in ovarian carcinoma GI-102. The specific bands of the transcript could not be detected in breast carcinoma GI101, lung carcinoma LX-1, and pancreatic adenocarcinoma GI-103. Interestingly, our data reveal that the transcript in colon adenocarcinoma CX-1 (well differentiated) is extremely high and that the transcript in colon adenocarcinoma GI-112 (poorly differentiated) is higher than that in the normal colon. This observation implies that $R a b 2 B$ may have a close relationship with colon tumors. Further studies will focus on the expression of $R a b 2 B$ protein and the role of human $R a b 2 B$.

Acknowledgments This research was funded by a grant from the Natural Science Foundation of China (30170345).

\section{References}

Baldini G, Hohl T, Lin HY, Lodish HF (1992) Cloning of a Rab3 isotype predominantly expressed in adipocytes. Proc Natl Acad Sci USA 89:5049-5052

Bourne HR, Sanders DA, McCormick F (1991) The GTPase superfamily: conserved structure and molecular mechanism. Nature 349:117127 
Culine S, Honore N, Tavitian A, Olofsson B (1992) Overexpression of the ras-related rab2 gene product in peripheral blood mononuclear cells from patients with hematological and solid neoplasms. Cancer Res 52:3083-3088

Culine S, Honore N, Closson V, Lang P, Bertoglio J, Tavitian A, Olofsson B (1993) A possible role for the Ras-related Rab2 protein in the immunological events associated with hematological malignancies. Nouv Rev Fr Hematol 35:41-44

Culine S, Honore N, Closson V, Droz JP, Extra JM, Marty M, Tavitian A, Olofsson B (1994) A small GTP-binding protein is frequently overexpressed in peripheral blood mononuclear cells from patients with solid tumours. Eur J Cancer 30:670-674
Lutcke A, Jansson S, Parton RG, Chavrier P, Valencia A, Huber LA, Lehtonen E, Zerial M (1993) Rab17, a novel small GTPase, is specific for epithelial cells and is induced during cell polarization. J Cell Biol 121:553-564

Nuoffer C, Balch WE (1994) GTPases: multifunctional molecular switches regulating vesicular traffic. Annu Rev Biochem 63:949-990

Pereiral-Leal JB, Seabra MC (2001) Evolution of the Rab family of small GTP-binding proteins. J Mol Biol 313:889-901

Tisdale EJ, Bourne JR, Khosravi-Far R, Der CJ, Balch WE (1992) GTP-binding mutants of rab1 and rab2 are potent inhibitors of vesicular transport from the endoplasmic reticulum to the Golgi complex. J Cell Biol 119:749-761 